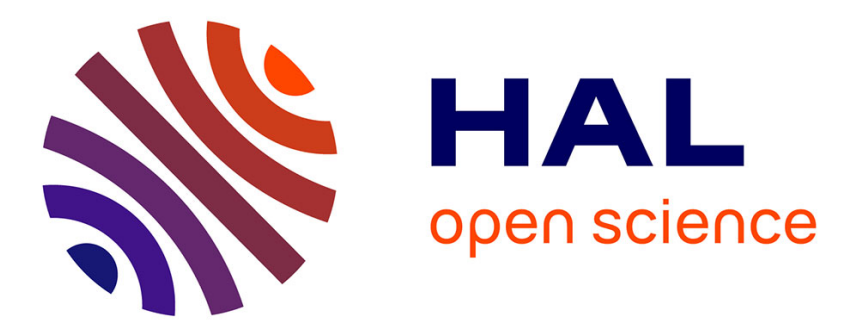

\title{
Analytical Modeling of an Inductor in a Magnetic Circuit for Pulsed Field Magnetization of HTS Bulks
}

Mohamed Elbaa, Kévin Berger, Bruno Douine, Mohamed Halit, El Hadj Ailam, Salah Eddine Bentridi

\section{- To cite this version:}

Mohamed Elbaa, Kévin Berger, Bruno Douine, Mohamed Halit, El Hadj Ailam, et al.. Analytical Modeling of an Inductor in a Magnetic Circuit for Pulsed Field Magnetization of HTS Bulks. IEEE Transactions on Applied Superconductivity, 2018, 28 (4), pp.8201306. 10.1109/TASC.2018.2809438 . hal-01592255v3

\author{
HAL Id: hal-01592255 \\ https://hal.science/hal-01592255v3
}

Submitted on 21 Feb 2018

HAL is a multi-disciplinary open access archive for the deposit and dissemination of scientific research documents, whether they are published or not. The documents may come from teaching and research institutions in France or abroad, or from public or private research centers.
L'archive ouverte pluridisciplinaire HAL, est destinée au dépôt et à la diffusion de documents scientifiques de niveau recherche, publiés ou non, émanant des établissements d'enseignement et de recherche français ou étrangers, des laboratoires publics ou privés. 


\title{
Analytical Modeling of an Inductor in a Magnetic Circuit for Pulsed Field Magnetization of HTS Bulks
}

\author{
Mohamed Elbaa, Kévin Berger, Bruno Douine, Mohamed Halit, El Hadj Ailam, and Salah Eddine Bentridi
}

\begin{abstract}
The development of bulk magnets based on REBCO type superconductors that can generate up to $17 \mathrm{~T}$ requires the implementation of a magnetization system, sometimes pulsed. Therefore, at least one coil around the High Temperature Superconducting (HTS) bulk is required. In this paper, we suggest an analytical approach in order to assess the magnetic vector potential and to compute the inductance of a coil having a rectangular crosssection and concentric with a magnetic circuit including an air-gap. On a proposed geometry, analytical results are compared with those obtained by using Finite Element Analysis (FEA). The gained analytical expressions give accurate results with an error less than $1 \%$ on the inductance value of the coil. The induced current density and trapped magnetic field in a HTS bulk during a Pulsed Field Magnetization (PFM) are also calculated using the proposed analytical expressions of the magnetic vector potential combined with an integral method. The achieved results show a remarkable match with those obtained with COMSOL.
\end{abstract}

Index Terms-Eddy currents, Electromagnets, Inductance, Modeling, Pulse measurements, Solenoids.

\section{INTRODUCTION}

$\mathbf{I}_{\mathrm{a} t}$ $\mathrm{N}$ electrical engineering as in physics, superconductors are widely used to obtain very strong magnetic fields. Superconducting bulk magnets made with (RE)BCO type superconductors are being considered to undergo pulsed magnetization and to reach very high magnetic flux density up to $17 \mathrm{~T}$ [1]. It is very important to estimate and evaluate the magnetic field components that strongly depends on the geometry of the used inductor, in the final aim to optimize the experimental configuration. In the design stage of the inductor needed for magnetizing the High Temperature Superconducting (HTS) bulks, modeling and simulation are highly recommended to perform necessary calculations of the different electromagnetic characteristics of the studied system, as the inductance of the coil. Sophisticated commercial software gives very accurate results by considering the nonlinearity of materials involved in the studied problem. However, these methods are computer time consuming and, mesh and all the physical quantities must be reassessed when the dimensions of the problem are changed.

Mohamed Elbaa, Kévin Berger and Bruno Douine are with the Group of Research in Electrical Engineering of Nancy, Faculty of Sciences and Technologies, University of Lorraine, 54506 Vandoeuvre-lès-Nancy, France (e-mail: name.surname@univ-lorraine.fr).

Mohamed Halit is with the Laboratoire Physique des Matériaux, University of Laghouat, Algeria.

Mohamed Elbaa, El Hadj Ailam and Salah Eddine Bentridi are with the Laboratoire de l'Energie et des Systèmes Intelligents, University of Khemis Miliana, Algeria.
Analytical models have been proposed since a long time for computing the magnetic field distribution of ironless circular coils [2]-[10] and more recently for an axisymmetric coil with an iron core of finite length [11]. Although all these methods give accurate results, they are not suitable to study circular coils placed in a magnetic circuit containing a large air-gap, for some of them because of the presence of the magnetic material, for some others due the air-gap.

In the present work, we propose to use analytical methods to achieve that task by the resolution of Laplace's and Poisson's equations. Such methods lead to meaningful solutions with very helpful physical insights at the design stage. They are useful tools during the optimization of the design since continuous derivatives are obtained from the analytical solutions. It allows us to calculate the magnetic vector potential $A$ in a non-closed magnetic circuit with an air-gap concentric with the coil. The gained analytical expressions are then used to calculate induced currents in a HTS bulk by means of the "Brandt's method" [12], [13]. An example of Pulsed Field Magnetization (PFM) of a bulk pellet placed in the air-gap is treated in Section VII.

An axisymmetric problem has to be considered here, allowing some simplifications and considerations to obtain field components over the different regions of interest. Four regions have to be considered, three free space regions (air) and one region with the coil conductor. Both Dirichlet and Neumann conditions are taken into account on the problem boundaries. In each region, the analytical solution is obtained as combination of Bessel and Struve functions, where coefficients are determined by the use of interface conditions between successive regions. Then, the distribution of magnetic vector potential and magnetic flux density are calculated with the help of MATLAB matrix inversion tool to determine the desired coefficients. Besides, a finite elements model is achieved with COMSOL Multiphysics software for the same definition of the problem. Analytical and Finite Element Analysis (FEA) results are also compared and discussed in what follows.

\section{PROBlem Formulation AND AsSUmptions}

The geometry of the studied problem is shown in Fig. 1. The regions $I, I I$ and $I V$ correspond to a free space filled with air, the region $I I$ corresponds to a circular coil of $N$ turns with rectangular cross section with inner radius $R_{2}$, outer radius $R_{3}$ and length $L=\left(Z_{4}-Z_{1}\right)$. We consider a uniform current density $J$ passing through the coil cross section in the $\theta$-direction. 


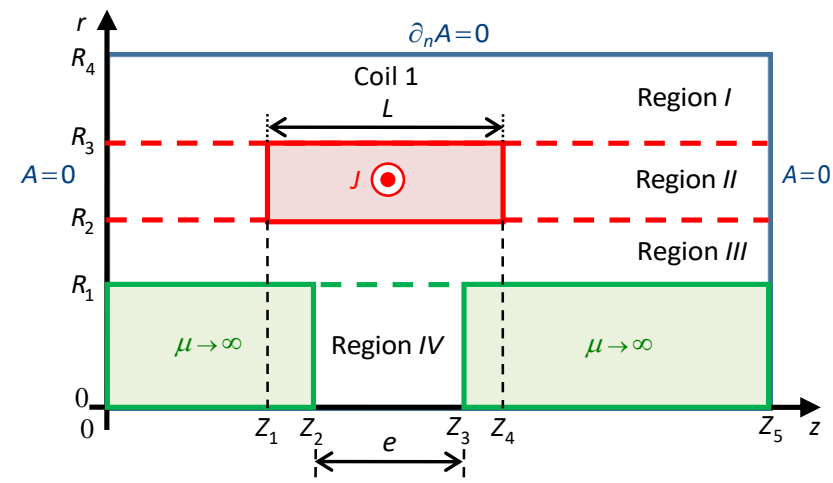

Fig. 1. Geometry of the studied problem. $L$ is the length of the coil while $e$ is the length of the air-gap of the magnetic circuit. Boundary with $\partial_{n} A=0$ represents a boundary with a ferromagnetic material, while boundaries with $A=0$ correspond to a magnetic isolation.

The coil is centered on the air-gap of length $e=\left(Z_{3}-Z_{2}\right)$ of the non-closed ferromagnetic circuit, located in the lower part as shown in Fig. 1. The whole system is limited in the axial direction by $z=0$ and $z=Z_{5}$, where the outer boundaries of the magnetic circuit must be chosen sufficiently far away from the calculation zone. The homogeneous Dirichlet boundary conditions are considered in regions $I, I I$ and $I I I$. However, homogeneous Neumann boundary conditions are used in regions $I V$.

Another approximation is employed in the modeling of this problem by assuming infinite permeability of the ferromagnetic circuit $\mu \rightarrow \infty$. This implies that the magnetic field is not calculated inside the ferromagnetic circuit. The interface conditions are then used to obtain the tangential component of magnetic field to be null.

The problem being axisymmetric, cylindrical coordinates are the most appropriated to the formulation of the magnetic vector potential, where only the $\theta$-component exists and it depends only on the $r$ and $z$ coordinates.

The expressions of magnetic vector potential are obtained with resolving a Poisson's equation in the coil region and a Laplace's equation in the other regions, as follows:

$\nabla^{2} A_{i}= \begin{cases}-\mu_{0} J & \text { for } i=I I \\ 0 & \text { for } i=\{I, I I I, \text { and } I V\}\end{cases}$

By using the separation of variables method [11], [14], we consider the general solution of (1) in Regions $I$ to $I V$.

Considering homogenous Dirichlet boundary conditions at $z=0$ and $z=Z_{5}$ in Regions $I, I I$ and $I I I$ and homogenous Neumann boundary conditions at $z=Z_{2}$ and $z=Z_{3}$ in Region $I V$ we have:

$$
\begin{cases}A_{i}(r, z=0)=0 \text { and } A_{i}\left(r, z=Z_{5}\right)=0 & \text { for } i=\{I, I I, I I I\} \\ \left.\partial_{z} A_{I V}\right|_{z=Z_{2}}=0 \text { and }\left.\partial_{z} A_{I V}\right|_{z=Z_{3}}=0 & \text { for } i=I V\end{cases}
$$

Moreover, the tangential component of the magnetic field is null at $r=R_{4}$

$$
\left.\partial_{r}\left(r A_{I}\right)\right|_{r=R_{4}}=0
$$

and the vector potential tends to zero when $r=0$

$$
A_{I V}(r=0, z)=0
$$

\section{Analytical Expressions of the MAGnetic Vector POTENTIAL}

Considering the boundary conditions (2), (3) and (4), the general solution of (1) can be expressed as:

$$
\begin{aligned}
& A_{I}(r, z)=\sum_{n=1}^{\infty} b_{n}^{I}\left(\frac{K_{0}\left(\alpha_{n} R_{4}\right)}{I_{0}\left(\alpha_{n} R_{4}\right)} I_{1}\left(\alpha_{n} r\right)+K_{1}\left(\alpha_{n} r\right)\right) \sin \left(\alpha_{n} z\right) \\
& A_{I I}(r, z)=\sum_{n=1}^{\infty}\left(\begin{array}{l}
\left.a_{n}^{I I} I_{1}\left(\alpha_{n} r\right)+b_{n}^{I I} K_{1}\left(\alpha_{n} r\right)\right) \sin \left(\alpha_{n} z\right) \\
-C_{n} L_{1}\left(\alpha_{n} r\right)
\end{array}\right) \\
& A_{I I I}(r, z)=\sum_{n=1}^{\infty}\left(a_{n}^{I I I} I_{1}\left(\alpha_{n} r\right)+b_{n}^{I I I} K_{1}\left(\alpha_{n} r\right)\right) \sin \left(\alpha_{n} z\right) \\
& A_{I V}(r, z)=A_{0} r+\sum_{k=1}^{\infty} a_{k}^{I V} I_{1}\left(\beta_{k} r\right) \cos \left(\beta_{k}\left(z-Z_{2}\right)\right)
\end{aligned}
$$

where $n$ and $k$ are positive integers, $\alpha_{n}=n \pi / Z_{5}$ and $\beta_{k}=$ $k \pi /\left(Z_{5}-Z_{2}\right)$ are the eigenvalues of the problem, $I_{1}$ and $K_{1}$ are respectively the modified Bessel functions of the first and second kind of order 1, and $L_{1}$ is the modified Struve function of order 1 .

$C_{n}=\frac{\mu_{0} \pi J_{n}}{2 \alpha_{n}^{2}}$, and $J_{n}=\frac{2 J}{n \pi}\left(\cos \left(\alpha_{n} Z_{1}\right)-\cos \left(\alpha_{n} Z_{4}\right)\right)$

$A_{0}$ is an integration constant due to the Neumann boundary conditions in Region $I V$. It can be determined by applying the condition of continuity of the magnetic vector potential at the interface between Region $I I I$ and Region $I V$.

$A_{I V}\left(r=R_{1}, z\right)=A_{I I I}\left(r=R_{1}, z\right)$, for $z \in\left[Z_{2}, Z_{3}\right]$

By integration in respect to the $z$ variable of (10) over the interval $\left[Z_{2}, Z_{3}\right]$, we obtain:

$A_{0}=\left(R_{1}\left(Z_{3}-Z_{2}\right)\right)^{-1} \sum_{n=1}^{\infty} m_{n}\left(a_{n}^{I I I} I_{1}\left(\alpha_{n} R_{1}\right)+b_{n}^{I I I} K_{1}\left(\alpha_{n} R_{1}\right)\right)$

with $m_{n}=\alpha_{n}^{-1}\left(\cos \left(\alpha_{n} Z_{2}\right)-\cos \left(\alpha_{n} Z_{3}\right)\right)$

$a_{n}^{I}, b_{n}^{I}, a_{n}^{I I}, b_{n}^{I I}, a_{n}^{I I I}, b_{n}^{I I I}$ and $a_{n}^{I V}$ are the integration constants. The relations between those integration constants are determined by applying the interface conditions between the different regions.

\section{INTERFACE CONDITIONS BETWEen THE REgIONS}

The interface conditions must satisfy the continuity of the radial component of the flux density and the continuity of the axial component of the magnetic field. The radial and axial flux density can be deduced from the magnetic vector potential by:

$B_{r}=-\partial_{z} A$ and $B_{z}=\frac{1}{r} \partial_{r}(r A)$

The interface conditions between Region $I$ and $I I$ at $r=R_{3}$ and region $I I$ and $I I I$ at $r=R_{2}$ lead to:

$\left\{\begin{array}{l}A_{I}\left(r=R_{3}, z\right)=A_{I I}\left(r=R_{3}, z\right) \\ \left.\partial_{r}\left(r A_{I}\right)\right|_{r=R_{3}}=\left.\partial_{r}\left(r A_{I I}\right)\right|_{r=R_{3}}\end{array}\right.$ 
$\left\{\begin{array}{l}A_{I I}\left(r=R_{2}, z\right)=A_{I I I}\left(r=R_{2}, z\right) \\ \left.\partial_{r}\left(r A_{I I}\right)\right|_{r=R_{2}}=\left.\partial_{r}\left(r A_{I I I}\right)\right|_{r=R_{2}}\end{array}\right.$

At the interface between Region $I I I$ and $I V$, specific conditions concerning the axial component of the magnetic field at $r=R_{2}$, piecewise defined, are applied around this interface because of the presence of the air-gap in the ferromagnetic circuit:

$$
\left.\partial_{r}\left(r A_{I I I}\right)\right|_{r=R_{1}}= \begin{cases}0, & z \in\left[0, Z_{2}\right] \\ \left.\partial_{r}\left(r A_{I V}\right)\right|_{r=R_{1}}, & z \in\left[Z_{2}, Z_{3}\right] \\ 0, & z \in\left[Z_{3}, Z_{5}\right]\end{cases}
$$

The continuity of the radial component of the flux density yields to:

$A_{I V}\left(r=R_{1}, z\right)=A_{I I I}\left(r=R_{1}, z\right), z \in\left[Z_{2}, Z_{3}\right]$

The coefficients of the regions $I$ to $I V$ are related to (12), (13), (14), (15), (16), by using the Fourier series method, and rewriting equations in matrix and vectors format:

$$
\begin{aligned}
& {\left[\begin{array}{cccccc}
\eta_{11} & \eta_{12} & \eta_{13} & 0 & 0 & 0 \\
\eta_{21} & \eta_{22} & \eta_{23} & 0 & 0 & 0 \\
0 & \eta_{32} & \eta_{33} & \eta_{34} & \eta_{35} & 0 \\
0 & \eta_{42} & \eta_{43} & \eta_{44} & \eta_{45} & 0 \\
0 & 0 & 0 & \eta_{54} & \eta_{55} & \eta_{56} \\
0 & 0 & 0 & \eta_{64} & \eta_{65} & \eta_{66}
\end{array}\right]\left[\begin{array}{c}
b_{n}^{I} \\
a_{n}^{I I} \\
b_{n}^{I I} \\
a_{n}^{I I I} \\
b_{n}^{I I I} \\
a_{n}^{I V}
\end{array}\right]=\left[\begin{array}{c}
-C_{n} L_{1}\left(\alpha_{n} R_{3}\right) \\
-C_{n} L_{0}\left(\alpha_{n} R_{3}\right) \\
-C_{n} L_{1}\left(\alpha_{n} R_{2}\right) \\
-C_{n} L_{0}\left(\alpha_{n} R_{2}\right) \\
0 \\
0
\end{array}\right]} \\
& \eta_{11}=\frac{K_{0}\left(\alpha_{n} R_{4}\right)}{I_{0}\left(\alpha_{n} R_{4}\right)} I_{1}\left(\alpha_{n} R_{3}\right)+K_{1}\left(\alpha_{n} R_{3}\right), \eta_{12}=-I_{1}\left(\alpha_{n} R_{3}\right), \\
& \eta_{13}=-K_{1}\left(\alpha_{n} R_{3}\right), \eta_{21}=\frac{K_{0}\left(\alpha_{n} R_{4}\right)}{I_{0}\left(\alpha_{n} R_{4}\right)} I_{0}\left(\alpha_{n} R_{3}\right)-K_{0}\left(\alpha_{n} R_{3}\right), \\
& \eta_{22}=-I_{0}\left(\alpha_{n} R_{3}\right), \eta_{23}=K_{0}\left(\alpha_{n} R_{3}\right), \eta_{32}=-I_{1}\left(\alpha_{n} R_{2}\right), \\
& \eta_{33}=-K_{1}\left(\alpha_{n} R_{2}\right), \eta_{34}=I_{1}\left(\alpha_{n} R_{2}\right), \eta_{35}=K_{1}\left(\alpha_{n} R_{2}\right), \\
& \eta_{42}=-I_{0}\left(\alpha_{n} R_{2}\right), \eta_{43}=K_{0}\left(\alpha_{n} R_{2}\right), \eta_{44}=I_{0}\left(\alpha_{n} R_{2}\right), \\
& \eta_{45}=-K_{0}\left(\alpha_{n} R_{2}\right), \eta_{54}=I_{1}\left(\alpha_{k} R_{1}\right) h_{n, k}-I_{0}\left(\alpha_{k} R_{1}\right) f_{n, k}, \\
& \eta_{55}=K_{1}\left(\alpha_{k} R_{1}\right) h_{n, k}+K_{0}\left(\alpha_{k} R_{1}\right) f_{n, k}, \eta_{56}=I_{0}\left(\beta_{k} R_{1}\right) g_{n, k}, \\
& \eta_{64}=I_{1}\left(\alpha_{n} R_{1}\right) g_{n, k}, \eta_{65}=K_{1}\left(\alpha_{n} R_{1}\right) g_{n, k}, \eta_{66}=-I_{1}\left(\beta_{k} R_{1}\right)
\end{aligned}
$$

with

$$
\begin{aligned}
& f_{n, k}=\left\{\begin{array}{l}
(-1)^{n+1} \frac{n^{2}}{n^{2}-k^{2}} \sin (k \pi), n \neq k \\
n \pi / 2, n=k
\end{array}\right. \\
& h_{n, k}=2 m_{n} m_{k}\left(R_{1}\left(Z_{3}-Z_{2}\right)\right)^{-1} \\
& g_{n, k}=\left\{\begin{array}{l}
\frac{2 \alpha_{n} \beta_{k}}{\left(\alpha_{n}^{2}-\beta_{k}^{2}\right)}\left(\begin{array}{l}
\cos \left(\alpha_{n} Z_{2}\right) \\
+(-1)^{k+1} \cos \left(\alpha_{n} Z_{3}\right)
\end{array}\right), \alpha_{n} \neq \beta_{k} \\
\frac{k \pi}{2} \sin \left(\beta_{k} Z_{2}\right)+\frac{1}{4}\left(\begin{array}{l}
\cos \left(\beta_{k} Z_{2}\right) \\
+(-1)^{k+1} \cos \left(\beta_{k} Z_{3}\right)
\end{array}\right), \alpha_{n}=\beta_{k}
\end{array}\right.
\end{aligned}
$$

Thus, we have to solve a system of six linear equations with six unknowns. By rewriting the above equations in matrix and vectors format, a numerical solution can be found by using mathematical software, e.g. the matrix inversion tool of MATLAB. It should be noted here that a numerical matrix inversion is required for the calculation of the unknown coefficients which could be used even in symbolic form.

\section{INDUCTANCE EXPRESSION}

The inductance of the coil is related to the total stored magnetic energy:

$W=\frac{1}{2} L I^{2}=\frac{1}{2} \int_{0}^{2 \pi} \int_{Z_{1}}^{Z_{4}} \int_{R_{2}}^{R_{3}} J A_{I I}(r, z) r d r d z d \theta$

Assuming that the current density is uniformly distributed over the whole cross section of the coil:

$I=J S / N$ with $S=\left(R_{3}-R_{2}\right)\left(Z_{4}-Z_{1}\right)$

where, $N$ and $I$ are respectively the number of turns in the coil and the electrical current in the wire. The magnetic vector potential is the one defined in the Region II of the problem.

Substituting (6) and (23) into (22) and integrating in respect to the $r, \theta$ and $z$ variables, the obtained analytical expression of the inductance is given by:

$$
L=2 \pi \frac{N^{2}}{J S^{2}} \sum_{n=1}^{\infty}\left\{\begin{array}{c}
\frac{\pi}{2 \alpha_{n}^{2}}\left(\begin{array}{l}
a_{n}^{I I}\left(R_{3} U\left(R_{3}\right)-R_{2} U\left(R_{2}\right)\right) \\
+b_{n}^{I I}\left(R_{3} V\left(R_{3}\right)-R_{2} V\left(R_{2}\right)\right) \\
-\mu_{0} J_{n} \frac{\alpha_{n}}{6 \pi}\left(R_{3}^{4} W\left(R_{3}\right)-R_{2}^{4} W\left(R_{2}\right)\right)
\end{array}\right) \\
\left(\cos \left(\alpha_{n} Z_{1}\right)-\cos \left(\alpha_{n} Z_{4}\right)\right)
\end{array}\right\}
$$

where $U(r), V(r)$ and $W(r)$ were defined in reference [11].

\section{Analytical Results AND COMParison with Finite ELEMENT SIMULATIONS}

Table I contains the values of the geometric parameters used in our problem. The outer boundaries in the axial direc-

TABLE I

GEOMETRIC PARAMETERS

\begin{tabular}{clc}
\hline \hline Symbol & \multicolumn{1}{c}{ Quantity } & Value \\
\hline$R_{1}$ & Lower edge of the domain in the radial direction & $0.030 \mathrm{~m}$ \\
$R_{2}$ & Inner radius of the coil & $0.080 \mathrm{~m}$ \\
$R_{3}$ & Outer radius of the coil & $0.120 \mathrm{~m}$ \\
$R_{4}$ & Upper edge of the domain in the radial direction & $0.150 \mathrm{~m}$ \\
$Z_{1}$ & Inner boundary of the coil & $0.200 \mathrm{~m}$ \\
$Z_{2}$ & Inner boundary of the air-gap & $0.225 \mathrm{~m}$ \\
$Z_{3}$ & Outer boundary of the air-gap & $0.275 \mathrm{~m}$ \\
$Z_{4}$ & Outer boundary of the coil & $0.300 \mathrm{~m}$ \\
$Z_{5}$ & Outer boundary of the domain in the axial direc- & $0.500 \mathrm{~m}$ \\
$L$ & tion & \\
$e$ & Axial length of the coil & $0.100 \mathrm{~m}$ \\
$N$ & Axial length of the air-gap & $0.050 \mathrm{~m}$ \\
$J$ & Number of turns of the coil & 1000 \\
$N_{\max }$ & Current density in the coil & $5 \mathrm{~A} / \mathrm{mm}^{2}$ \\
\hline
\end{tabular}




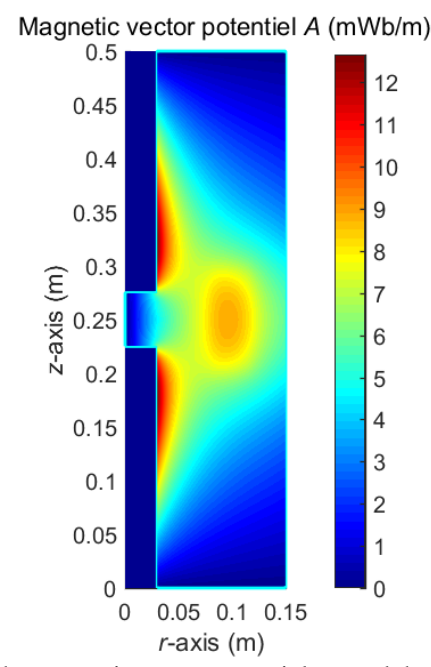

Fig. 2. Distribution of the magnetic vector potential around the air-gap.
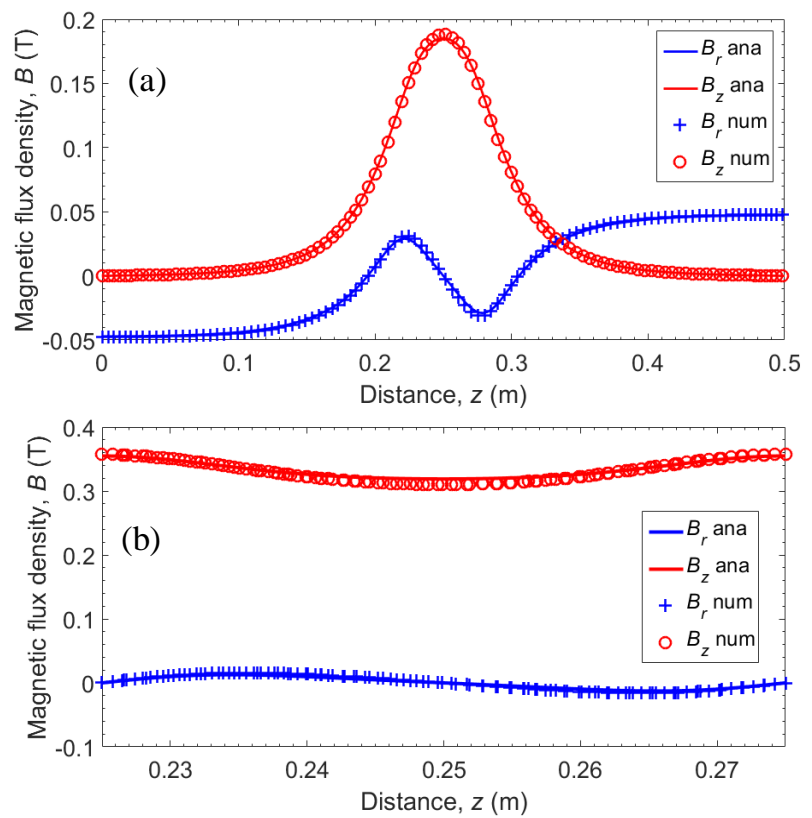

Fig. 3. Radial and axial components of the magnetic flux density $B_{r}$ and $B_{z}$ along the $z$-axis, (a) in the Region $I I I$ at $r=\left(R_{1}+R_{2}\right) / 2$ and (b) in the air-gap at $r=R_{1} / 2$.

tion are chosen such that their values do not affect the obtained results. The analytical solutions in all the regions have been computed with a finite number of harmonic terms equal to 50 .

In order to validate our analytical results, we have to compare them with those obtained by using FEA led on COMSOL Multiphysics software. The mesh size and the requested precision are chosen so that the quantities derived from the FEA serve as a reference. Obviously, the same geometric parameters as those defined in Table I are used. Homogeneous Dirichlet and Neumann boundaries conditions are also considered in simulations as with the analytical model.

Fig. 2 shows the distribution of magnetic vector potential of the defined problem when the air-gap is centered with the coil. The radial and the axial components of the magnetic flux density along the $z$-axis in Region $I I I$ at $r=\left(R_{1}+R_{2}\right) / 2$ are plotted and compared with the numerical results in Fig. 3(a). Fig. 3(b)

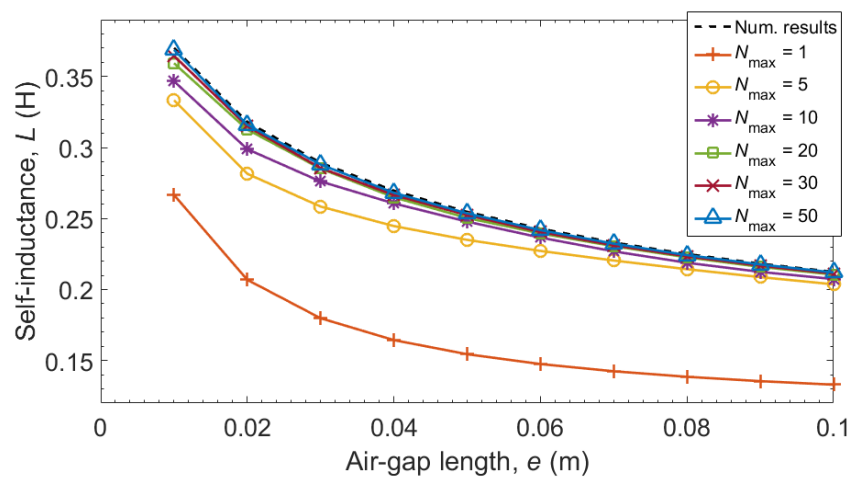

Fig. 4. Self-inductance of the coil using (24) with an air-gap length varying from $0.01 \mathrm{~m}$ to $0.05 \mathrm{~m}$. Comparison between the numerical values obtained with COMSOL and the analytical results for different number of harmonic terms from 1 to 50 .

also shows $B_{r}$ and $B_{z}$ along the $z$-axis but in the air-gap, at $r=R_{1} / 2$.

From Fig. 3 we can clearly observe that the results obtained by the analytical method are very closer to those obtained by the FEA software. A small discrepancy between the analytical and numerical results still exists. The maximal discrepancy is lower than $3 \%$ for the $B_{z}$ component and around $10 \%$ for $B_{r}$. These discrepancies can be explained by the limitations of the analytic development, in particular the numerical errors linked to the matrix inversion that generates errors during the evaluation of the coefficients. Indeed, the use of modified Bessel functions leads to badly conditioned matrix.

Finally, Fig. 4 shows the self-inductance of the coil using (24) with an air-gap length varying from $0.01 \mathrm{~m}$ to $0.1 \mathrm{~m}$. A comparison is made between the numerical values obtained with COMSOL and the analytical results for different number of harmonic terms from 1 to 50. Obviously, the inductance decreases with the increase of the air-gap length. The higher the number of harmonic terms, the closer is the solution to the reference value obtained numerically. The maximal error is of $39.37 \%, 11.47 \%, 6.35 \%, 2.92 \%, 1.52 \%$ and $0.75 \%$ for a number of harmonic terms of $1,5,10,20,30,40$, and 50 respectively. We can conclude here that the proposed formula is correct and efficient. Moreover, only 10 terms are enough to achieve less than $10 \%$ of error.

\section{EXAMPLE OF PFM OF A BULK HTS}

A bulk pellet with an outer radius $R_{11}=20 \mathrm{~mm}$ and a height $\left(Z_{33}-Z_{22}\right)=15 \mathrm{~mm}$ is placed in the middle of the air-gap. The analytical expression (8) of the applied magnetic vector poten-

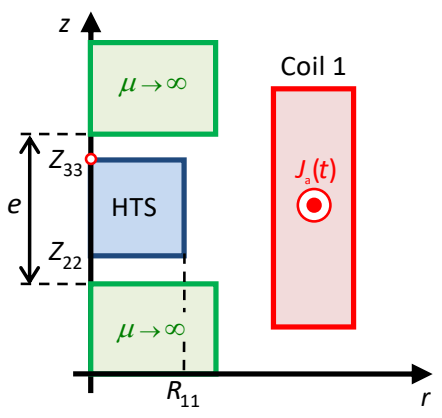

Fig. 5. Location of the bulk HTS in the problem described in Fig. 1, corresponding to $r \in\left[0, R_{11}\right]$ and $z \in\left[Z_{22}, Z_{33}\right]$. 
tial $A_{I V}(r, z, t)$ is used to compute the induced currents inside the bulk HTS. Fig. 5 shows the area of interest with the $z$-axis positioned vertically. To be realistic, the axial length $e$ of the air-gap is now of $20 \mathrm{~mm}$ and the current density $J_{\mathrm{a}}$ in the coil depends on time. This current density is assumed to be produced by the discharge of a capacitor bank [15], [16] as:

$$
J_{\mathrm{a}}(t)=J_{\text {max }}(t / \tau) \exp (1-(t / \tau))
$$

with $J_{\max }=50 \mathrm{~A} / \mathrm{mm}^{2}$ and $\tau=1 \mathrm{~ms}$.

The induced current density $J$ in the bulk HTS is calculated by means of a homemade code on MATLAB by using the well-known "Brandt's method" developed in 1998 for disks and cylinders in an axial magnetic field [12], [13]. In summary, the following equation must be satisfied:

$$
\frac{d J\left(\mathbf{r}_{2}, t\right)}{d t}=\mu_{0}^{-1} \int_{0}^{R_{11}} \int_{Z_{22}}^{Z_{33}} Q_{\mathrm{cyl}}^{-1}\left(\mathbf{r}_{2}, \mathbf{r}_{2}^{\prime}\right)\left(E(J)+\frac{d A_{I V}\left(\mathbf{r}_{2}^{\prime}, t\right)}{d t}\right) d r^{\prime} d z^{\prime}
$$

with $\mathbf{r}_{2}=(r, z)$ the coordinates of the bulk HTS, $Q_{\text {cyl }}$ is the kernel obtained by integrating the 3D Green function of the Laplace equation and taking into account the vicinity of the ferromagnetic material with an infinite permeability, by means of the method of images. $E(J)$ is the relationship between the electrical field $E$ and the current density $J$ used to describe the superconducting behavior of the bulk. In our case, the electrical behavior of the HTS bulk is defined by the power law [15]:

$$
E(J)=\left(E_{\mathrm{c}} / J_{\mathrm{c}}\right)\left(|J| / J_{\mathrm{c}}\right)^{n-1} J
$$

with $E_{\mathrm{c}}=1 \mu \mathrm{V} / \mathrm{cm}, n=30$ and a constant critical current density $J_{\mathrm{c}}=100 \mathrm{~A} / \mathrm{mm}^{2}$. The resolution of the stiff Ordinary Differential Equation (26) is performed using available solvers in MATLAB as "ode15s" or "ode23t".

Fig. 6 shows the time evolution of the applied magnetic field $B_{\mathrm{a}}$ and the trapped magnetic field $B_{\text {trap }}$ on the top surface of the bulk HTS, at $r=0$ and $z=Z_{33}$ on Fig. 5. The results obtained through our method are compared with those achieved using an $\mathbf{H}$-formulation in COMSOL [17], [18]. An almost perfect match within the results is observed. The maximal magnetic flux density applied to the bulk HTS is about 7.51 T and the trapped magnetic flux density on its top surface is $2.09 \mathrm{~T}$ at $t=10 \mathrm{~ms}$. The discrepancy between the results is less than 3\% throughout the PFM process.

The normalized current density $J / J_{\mathrm{c}}$ in the middle plane of the bulk HTS is plotted versus time on Fig. 7 for several values of radii, $r=\{0,4.7,9.5,15.3,20\} \mathrm{mm}$. The results obtained with our method are close to those of COMSOL. There are slight discrepancies, in particular for the case $r=0$. This radius value is singular and we cannot determine which of the results is correct or not. In any case, the value of the current density on the axis does not contribute much in terms of trapped magnetic field. A $t=5 \mathrm{~ms}$, the current density in the bulk HTS is within the range of 1.1 to $1.5 \times J_{\mathrm{c}}$.

In this paragraph, we will give readers some approximated values of calculation time for this problem solved on an Intel® Core ${ }^{\mathrm{TM}}$ i7-6700 CPU @ 3.40 GHz (Turbo Boost $4.00 \mathrm{GHz}$ ), 32 GB RAM, with MATLAB R2016b running under Windows 1064 bits:

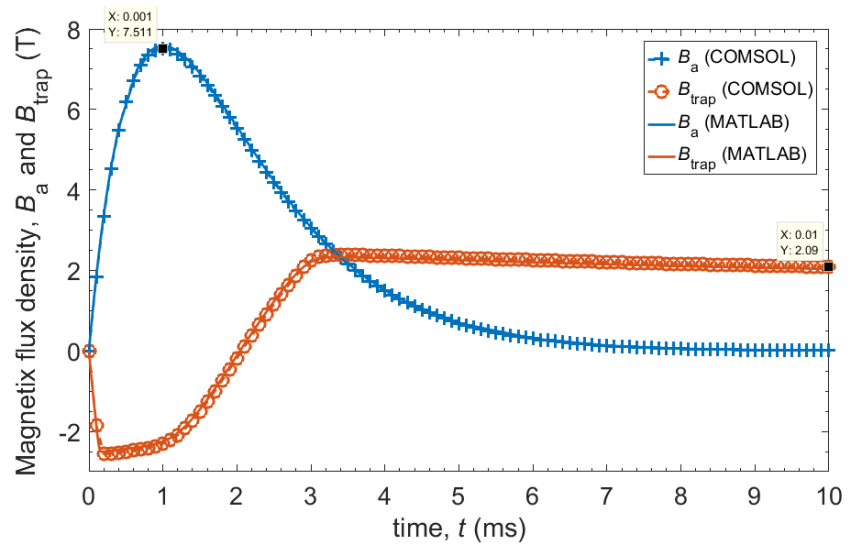

Fig. 6. Time evolution of the applied magnetic field $B_{\mathrm{a}}$ and the trapped magnetic field $B_{\text {trap }}$ on the top surface of the bulk HTS, at $r=0$ and $z=Z_{33}$.

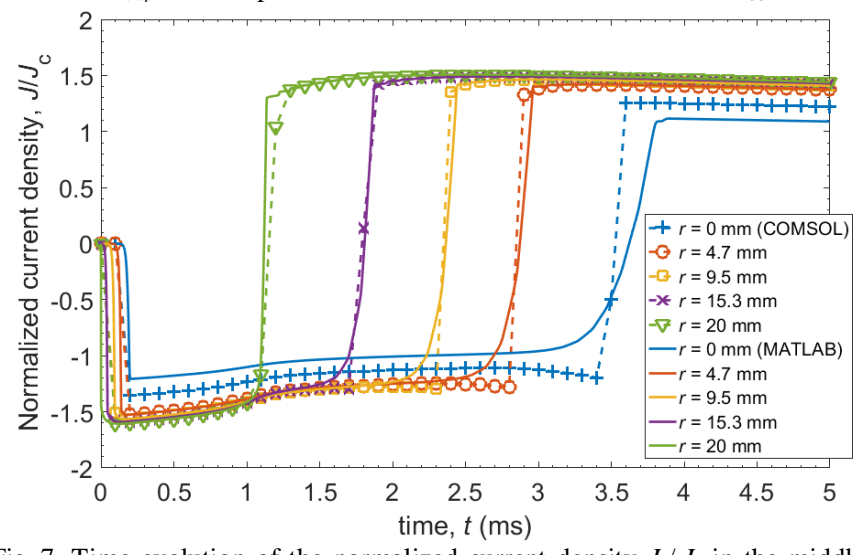

Fig. 7. Time evolution of the normalized current density $J / J_{\mathrm{c}}$ in the middle plane of the bulk HTS for $r=\{0,4.7,9.5,15.3,20\} \mathrm{mm}$.

$\rightarrow 2 \mathrm{~s}$ are required in order to obtain the coefficients $a_{n}^{I}, b_{n}^{I}, a_{n}^{I I}, b_{n}^{I I}, a_{n}^{I I I}, b_{n}^{I I I}$ and $a_{n}^{I V}$ by inverting the matrix (17) with 90 harmonic terms,

$\rightarrow 60 \mathrm{~s}$ are necessary to build all the topological matrices as $Q_{\text {cyl }}$ and others to calculate $B_{r}$ and $B_{z}$,

$\rightarrow 280 \mathrm{~s}$, less than $5 \mathrm{~min}$, are required to solve (26) using "ode23t" within an irregular mapped mesh $N_{r} \times N_{z}=30 \times 21$.

\section{CONCLUSION}

In this paper, we have developed analytical formulas complementary to those proposed in [11] to calculate the inductance of a coil having a rectangular cross-section and concentric with an iron core including an air-gap. We have shown that analytical methods provide a very good match with numerical ones with errors less than $10 \%$ for 10 harmonic terms, which is enough in the design stage. For PFM processes with capacitor bank, the inductance value is relevant since it defines the maximal value of current. The gained analytical expressions of the magnetic vector potential have been used to calculate induced currents in a bulk HTS by means of the "Brandt's method". The time evolution of various quantities shows an excellent match with the results obtained by a more classical FEA software. An overall discrepancy less than 3\% is obtained on the trapped magnetic flux density. The computation times are attractive and similar to those obtained with FEA. 


\section{ACKNOWLEDGMENT}

The authors would like to thank Thierry Lubin and Alexandre Colle for their useful discussions on this topic.

\section{REFERENCES}

[1] M. Tomita and M. Murakami, "High-temperature superconductor bulk magnets that can trap magnetic fields of over 17 tesla at 29 K," Nature, vol. 421, no. 6922, pp. 517-520, Jan. 2003.

[2] L. Urankar, "Vector potential and magnetic field of current-carrying finite arc segment in analytical form, Part III: Exact computation for rectangular cross section," IEEE Trans. Magn., vol. 18, no. 6, pp. 18601867, Nov. 1982.

[3] M. W. Garrett, "Calculation of Fields, Forces, and Mutual Inductances of Current Systems by Elliptic Integrals," J. Appl. Phys., vol. 34, no. 9, pp. 2567-2573, Sep. 1963.

[4] D. Yu and K. Han, "Self-inductance of air-core circular coils with rectangular cross section," IEEE Trans. Magn., vol. 23, no. 6, pp. 39163921, Nov. 1987.

[5] A. Rezzoug, J. P. Caron, and F. M. Sargos, "Analytical calculations of flux induction and forces of thick coils with finite length," IEEE Trans. Magn., vol. 28, no. 5, pp. 2250-2252, Sep. 1992.

[6] S. I. Babic and C. Akyel, "Improvement in the Analytical Calculation of the Magnetic Field Produced by Permanent Magnet Rings," Prog. Electromagn. Res. C, vol. 5, pp. 71-82, 2008.

[7] R. Ravaud, G. Lemarquand, V. Lemarquand, S. I. Babic, and C. Akyel, "Mutual Inductance and Force Exerted Between Thick Coils," Prog. Electromagn. Res., vol. 102, pp. 367-380, 2010.

[8] J. T. Conway, "Inductance Calculations for Circular Coils of Rectangular Cross Section and Parallel Axes Using Bessel and Struve Functions," IEEE Trans. Magn., vol. 46, no. 1, pp. 75-81, Jan. 2010.

[9] B. L. J. Gysen, K. J. Meessen, J. J. H. Paulides, and E. A. Lomonova, "General Formulation of the Electromagnetic Field Distribution in Ma- chines and Devices Using Fourier Analysis," IEEE Trans. Magn., vol. 46, no. 1, pp. 39-52, Jan. 2010.

[10] S. Babic, F. Sirois, C. Akyel, G. Lemarquand, V. Lemarquand, and R. Ravaud, "New Formulas for Mutual Inductance and Axial Magnetic Force Between a Thin Wall Solenoid and a Thick Circular Coil of Rectangular Cross-Section," IEEE Trans. Magn., vol. 47, no. 8, pp. 20342044, Aug. 2011.

[11] T. Lubin, K. Berger, and A. Rezzoug, "Inductance and Force Calculation for Axisymmetric Coil Systems Including an Iron Core of Finite Length," Prog. Electromagn. Res. B, vol. 41, pp. 377-396, Jun. 2012.

[12] E. H. Brandt, "Superconductor disks and cylinders in an axial magnetic field. I. Flux penetration and magnetization curves," Phys. Rev. B, vol. 58, no. 10, pp. 6506-6522, Sep. 1998.

[13] E. H. Brandt, "Superconductor disks and cylinders in an axial magnetic field: II. Nonlinear and linear ac susceptibilities," Phys. Rev. B, vol. 58, no. 10 , pp. 6523-6533, Sep. 1998.

[14] T. Lubin, S. Mezani, and A. Rezzoug, "Exact Analytical Method for Magnetic Field Computation in the Air Gap of Cylindrical Electrical Machines Considering Slotting Effects," IEEE Trans. Magn., vol. 46, no. 4, pp. 1092-1099, Apr. 2010.

[15] K. Berger, J. Lévêque, D. Netter, B. Douine, and A. Rezzoug, "Influence of Temperature and/or field dependencies of the E-J power law on Trapped magnetic field in bulk YBaCuO," IEEE Trans. Appl. Supercond., vol. 17, no. 2, pp. 3028-3031, 2007.

[16] B. Gony, K. Berger, B. Douine, M. R. Koblischka, and J. Lévêque, "Improvement of the Magnetization of a Superconducting Bulk using an Iron Core," IEEE Trans. Appl. Supercond., vol. 25, no. 3, pp. 1-4, Jun. 2015.

[17] R. Brambilla, F. Grilli, and L. Martini, "Development of an edgeelement model for AC loss computation of high-temperature superconductors," Supercond. Sci. Technol., vol. 20, no. 1, p. 16, 2007.

[18] K. Berger et al., "Benchmark on the 3D Numerical Modeling of a Superconducting Bulk," in 21st International Conference on the Computation of Electromagnetic Fields (Compumag 2017), Daejeon, South Korea, 2017, p. (ID 110). 